\title{
The convergence of $(p, q)$-Bernstein operators for the Cauchy kernel with a pole via divided difference
}

\author{
Faisal Khan', Mohd Saif², Aiman Mukheimer ${ }^{3}$ and M. Mursaleen ${ }^{1 *}$
}

\section{"Correspondence:}

mursaleenm@gmail.com

'Department of Mathematics, Aligarh Muslim University, Aligarh, India

Full list of author information is available at the end of the article

\section{Springer}

\begin{abstract}
In this paper, some qualitative approximation results for the $(p, q)$-Bernstein operators $B_{p, q}^{n}(f ; x)$ are obtained for the Cauchy kernel $\frac{1}{x-\alpha}$ with a pole $\alpha \in[0,1]$ for $q>p>1$. The main focus lies in the study of behavior of operators $B_{p, q}^{n}(f ; x)$ for the function $f_{m}(x)=\frac{1}{x-p^{m} q^{-m}}, x \neq p^{m} q^{-m}$ and $f_{m}\left(p^{m} q^{-m}\right)=a, a \in \mathbb{R}$ and the extra parameter $p$ provides flexibility for the approximation.

MSC: $41 \mathrm{~A} 10 ; 41 \mathrm{~A} 25 ; 41 \mathrm{~A} 36$

Keywords: $(p, q)$-integer; $(p, q)$-Bernstein operator; Convergence; Approximation of unbounded function; Cauchy kernel
\end{abstract}

\section{Introduction and preliminaries}

The uniform convergence of a sequence of operators to a continuous function was introduced by Bohman [9] and Korovkin [16]. Through $q$-calculus various modifications of Bernstein operators [7] have been studied so far [10, 18, 31]. The $(p, q)$-integers are the generalization of the $q$-integers, which has an important role in the representation theory of quantum calculus in the physics literature. Recently, the approximation by the $(p, q)$ analog of a positive linear operator has become an active area of research. For the theory and numerical implementations of the $(p, q)$-analog of Bernstein operators introduced by Mursaleen et al. [22] and other $(p, q)$-analogs, the reader may refer to [1-5, 11-15, 19-21] and [32]. For most recent work on the $(p, q)$-approximation we refer to [8, 24, 26].

The $(p, q)$-integer, $(p, q)$-binomial expansion and the $(p, q)$-binomial coefficients are defined by

$$
\begin{aligned}
& {[m]_{p, q}:=} \frac{p^{m}-q^{m}}{p-q}, \quad m=0,1,2, \ldots, p>q \geq 1, \\
&(a+b)_{p, q}^{m}::=(a+b)(p a+q b)\left(p^{2} a+q^{2} b\right) \cdots\left(p^{m-1} a+q^{m-1} b\right) \\
&=\sum_{r=0}^{k} p^{\frac{(m-r)(m-r-1)}{2}} q^{\frac{r(r-1)}{2}}\left[\begin{array}{c}
m \\
r
\end{array}\right]_{p, q} a^{r}, \\
& {\left[\begin{array}{c}
m \\
r
\end{array}\right]_{p, q}:=\frac{[m]_{p, q} !}{[r]_{p, q} ![m-r]_{p, q} !} . }
\end{aligned}
$$

(c) The Author(s) 2019. This article is distributed under the terms of the Creative Commons Attribution 4.0 International License (http://creativecommons.org/licenses/by/4.0/), which permits unrestricted use, distribution, and reproduction in any medium, provided you give appropriate credit to the original author(s) and the source, provide a link to the Creative Commons license, and indicate if changes were made. 
It can easily be verified by induction that

$$
(1+a)(p+q a)\left(p^{2}+q^{2} a\right) \cdots\left(p^{n-1}+q^{n-1} a\right)=\sum_{r=0}^{k} p^{\frac{(m-r)(m-r-1)}{2}} q^{\frac{r(r-1)}{2}}\left[\begin{array}{c}
m \\
r
\end{array}\right]_{p, q} a^{r} .
$$

The $(p, q)$-analog of Euler's identity is defined by

$$
\prod_{s=0}^{m-1}\left(p^{s}-q^{s} a\right):=\sum_{k=0}^{m} p^{\frac{(m-k)(m-k-1)}{2}} q^{\frac{k(k-1)}{2}}\left[\begin{array}{c}
m \\
k
\end{array}\right]_{p, q} a^{k} .
$$

Let $f:[0,1] \longrightarrow \mathbb{R}$ and $q>p>1$. The $(p, q)$-Bernstein operators [22] of $f$ is defined as

$$
B_{p, q}^{n}(f ; x):=\sum_{k=0}^{n} f\left(\frac{[k]_{p, q}}{p^{k-n}[n]_{p, q}}\right) p_{n, k}(p, q ; x), \quad n \in \mathbb{N}
$$

where the polynomial $p_{n, k}(p, q ; x)$ is given by

$$
p_{n, k}(p, q ; x)=\frac{1}{p^{\frac{n(n-1)}{2}}}\left[\begin{array}{l}
n \\
k
\end{array}\right]_{p, q} p^{\frac{k(k-1)}{2}} x^{k} \prod_{s=0}^{n-k-1}\left(p^{s}-q^{s} x\right), \quad x \in[0,1], 0<q<p<1 .
$$

For $p=1, B_{p, q}^{n}(f ; x)$ turns into the $q$-Bernstein operator. We have

$$
B_{p, q}^{n}(f ; 0)=f(0), \quad B_{p, q}^{n}(f ; 1)=f(1), \quad n \in \mathbb{N} .
$$

The following $(p, q)$-difference form of Bernstein operators [25] is given by

$$
B_{p, q}^{n}(f ; x):=\sum_{r=0}^{n} \lambda_{p, q}^{n} f\left[0, \frac{p^{n-1}[1]_{p, q}}{[n]_{p, q}}, \ldots, \frac{p^{n-r}[r]_{p, q}}{[n]_{p, q}}\right] x^{r}
$$

where $f\left[x_{0}, x_{1}, \ldots, x_{n}\right]$ indicates the $n$th order divided difference of $f$ with pairwise distinct node, that is,

$$
\begin{aligned}
& f\left[x_{0}\right]=f\left(x_{0}\right), \quad f\left[x_{0}, x_{1}\right]=\frac{f\left(x_{1}\right)-f\left(x_{0}\right)}{x_{1}-x_{0}}, \\
& f\left[x_{0}, x_{1}, \ldots, x_{n}\right]=\frac{f\left[x_{1}, \ldots, x_{n}\right]-f\left[x_{0}, \ldots, x_{n-1}\right]}{\left[x_{n}-x_{0}\right]}
\end{aligned}
$$

and $\lambda_{p, q}^{n}$ is given by

$$
\begin{aligned}
\lambda_{p, q}^{n} & =\left[\begin{array}{l}
n \\
r
\end{array}\right]_{p, q} \frac{[r]_{p, q} !}{[n]_{p, q}^{r}} p^{\frac{(n-r)(n-r-1)}{2}} q^{\frac{r(r-1)}{2}} \\
& =\left(1-\frac{p^{n-1}[1]_{p, q}}{[n]_{p, q}}\right)\left(1-\frac{p^{n-2}[2]_{p, q}}{[n]_{p, q}}\right) \cdots\left(1-\frac{p^{n-r+1}[r-1]_{p, q}}{[n]_{p, q}}\right),
\end{aligned}
$$

and $\lambda_{p, q}^{0}=\lambda_{p, q}^{1}=1,0 \leq \lambda_{p, q}^{n} \leq 1, r=0,1, \ldots, n$.

In this paper, some qualitative approximation results for the $(p, q)$-Bernstein operators $B_{p, q}^{n}(f ; x)$ have been obtained for the Cauchy kernel $\frac{1}{x-\alpha}$ with a pole $\alpha \in[0,1]$ for $q>p>1$. 
The main focus lies in the study of behavior of operators $B_{p, q}^{n}(f ; x)$ for the function $f_{m}(x)=$ $\frac{1}{x-p^{m} q^{-m}}, x \neq p^{m} q^{-m}$ and $f_{m}\left(p^{m} q^{-m}\right)=a, a \in \mathbb{R}$ and the extra parameter $p$ provides flexibility for the approximation.

The time scale $\mathbb{J}_{p, q}$ for $q>p>1$ is denoted and defined as

$$
\mathbb{J}_{p, q}=\{0\} \cup\left\{p^{k} q^{-k}\right\}_{k=0}^{\infty} .
$$

Here, we consider the $(p, q)$-Bernstein operators with the Cauchy kernel $\frac{1}{x-\alpha}, \alpha \in[0,1]$. The previously obtained results [27-30] lead to the following conclusions.

- If $\alpha=0$, that is, $f(x)=\frac{1}{x}, x \neq 0$ and $f(0)=a$, then, for $q \geq 2$,

$$
\lim _{n \rightarrow \infty} B_{p, q}^{n}(f ; x)= \begin{cases}f(x), & x \in \mathbb{J}_{p, q} \\ \infty, & x \notin \mathbb{J}_{p, q}\end{cases}
$$

- If $\alpha \in \mathbb{J}_{p, q} \backslash[0,1]$ that is $f(x)=\frac{1}{(x-\alpha)}$ if $x \neq \alpha$ and $f(\alpha)=a$, then

$$
\lim _{n \rightarrow \infty} B_{p, q}^{n}(f ; x)=f(x), \quad x \in \mathbb{J}_{p, q}
$$

Furthermore, as $n \rightarrow \infty, B_{p, q}^{n}(f ; x) \rightarrow f(x)$ uniformly on any compact subset of $(-\alpha, \alpha)$ and $B_{p, q}^{n}(f ; x) \rightarrow \infty$ for $|x|>\alpha, x \notin \mathbb{J}_{p, q}$. Therefore, it is left to examine the case $\alpha \in \mathbb{J}_{p, q} \backslash\{0\}$ which is exactly the subject of the present paper. Let the function $f_{m}: \mathbb{R} \rightarrow \mathbb{R}$ be defined by

$$
f_{m}(x)=\left\{\begin{array}{ll}
\frac{1}{\left(x-p^{m} q^{-m}\right)^{j}}, & x \in \mathbb{R} \backslash\left\{p^{m} q^{-m}\right\}, \\
a, & x=p^{m} q^{-m},
\end{array} \quad m \in \mathbb{N}_{0}, a \in \mathbb{R} .\right.
$$

\section{Some auxiliary results}

In this section, we prove some important lemmas.

Lemma 2.1 For the function $f_{m}$ defined by (1.8), we have

(a) for $m \in \mathbb{N}$,

$$
\lim _{n \rightarrow \infty} B_{p, q}^{n}\left(f_{m} ; p^{j} q^{-j}\right)=f_{m}\left(p^{j} q^{-j}\right), \quad j \in \mathbb{N}_{0} \backslash\{m, m+1\} .
$$

Besides,

$$
\begin{aligned}
& \lim _{n \rightarrow \infty} B_{p, q}^{n}\left(f_{m} ; p^{m} q^{-m}\right)=-\infty, \quad \text { and } \\
& \lim _{n \rightarrow \infty} B_{p, q}^{n}\left(f_{m} ; p^{-(m+1)} q^{-(m+1)}\right)=f_{m}\left(p^{m+1} q^{-(m+1)}\right)-\frac{p^{-m} q^{m}[m+1]_{p, q}}{p^{-1}(q-1)[m]_{p, q}}
\end{aligned}
$$

(b) For $m=0$,

$$
\lim _{n \rightarrow \infty} B_{p, q}^{n}\left(f_{0} ; p^{j} q^{-j}\right)=f_{0}\left(p^{j} q^{-j}\right), \quad j \in \mathbb{N}_{0}
$$

i.e., $B_{p, q}^{n}\left(f_{0} ; \cdot\right)$ approximates $f_{0}$ on $\mathbb{J}_{p, q}$. 
This describes the behavior of $B_{p, q}^{n}\left(f_{m} ; \cdot\right)$ on the time scale $\mathbb{J}_{p, q}$.

Proof (a) From (1.2), we can easily see that $p_{n, n-k}\left(p, q ; p^{j} q^{-j}\right)=0$ for $k>j$, whence

$$
B_{p, q}^{n}\left(f ; p^{-j} q^{j}\right)=\sum_{k=0}^{\min \{k, j\}} f\left(\frac{[n-k]_{p, q}}{[n]_{p, q}}\right) p_{n, n-k}\left(p, q ; p^{j} q^{-j}\right)
$$

Besides

$$
\lim _{n \rightarrow \infty} p_{n, n-k}\left(p, q ; p^{j} q^{-j}\right)=\delta_{k, j} \quad \text { and } \quad \lim _{n \rightarrow \infty} \frac{[n-k]_{p, q}}{[n]_{p, q}}=p^{k} q^{-k}
$$

Thus, $\lim _{n \rightarrow \infty} f_{m}\left(\frac{[n-k]_{p, q}}{[n]_{p, q}}\right) p_{n, n-k}\left(p, q ; p^{j} q^{-j}\right)=f_{m}\left(p^{k} q^{-k}\right) \delta_{j, k}$ for all $k \neq m$.

Now by easy calculation, we have

$$
\lim _{n \rightarrow \infty} f\left(\frac{[n-k]_{p, q}}{[n]_{p, q}}\right) p_{n, n-k}\left(p, q ; p^{j} q^{-j}\right)= \begin{cases}-\infty, & j=m, \\ -\frac{p^{-m} q^{m}[m+1]_{p, q}}{p^{-1}(q-1)[m]_{p, q}}, & j=m+1, \\ 0, & \geq m+2,\end{cases}
$$

and combining with (2.1) and (2.2) yields the result.

(b) It can be obtained easily from (1.3) and (2.2) as $f_{0}$ is continuous at all points $p^{j} q^{-j}$, $j \in \mathbb{N}$.

The next lemma is related to the coefficient of $B_{p, q}^{n}\left(f_{0} ; \cdot\right)$.

Lemma 2.2 Let $f_{m}$ be a function as in (1.8). If $B_{p, q}^{n}\left(f_{m} ; x\right)=\sum_{k=0}^{n} C_{k, n}^{p, q} x^{k}$ and $\frac{[k]_{p, q}}{[n]_{p, q}} \neq p^{m} q^{-m}$ for $k=0,1,2, \ldots, n$, then

$$
C_{k, n}^{p, q}=-\frac{\lambda_{k, n}^{p, q} p^{-m(k+1)} q^{m(k+1)}}{\left(1-\frac{p^{n-m-1} q^{m}[1]_{p, q}}{[n]_{p, q}}\right)\left(1-\frac{p^{n-m-2} q^{m}[2]_{p, q}}{[n]_{p, q}}\right) \cdots\left(1-\frac{p^{n-m-k} q^{m}[k]_{p, q}}{[n]_{p, q}}\right)},
$$

where $\lambda_{k, n}^{p, q}$ are given by (1.5).

Proof Consider $f_{m}(z)=\frac{1}{z-p^{m} q^{-m}}$, which is analytic function in $\mathbb{C} \backslash\left\{p^{m} q^{-m}\right\}$. It is well known that [17] the $k$ th order divided difference of $f$ can be expressed as

$$
f\left[x_{0}, x_{1}, \ldots, x_{k}\right]=\frac{1}{2 \pi i} \oint_{\mathcal{L}} \frac{f(\zeta) d \zeta}{\left(\zeta-x_{0}\right)\left(\zeta-x_{1}\right) \cdots\left(\zeta-x_{k}\right)}
$$

where $\mathcal{L}$ is contour encircling $x_{0}, \ldots, x_{k}$ and $f$ is assumed to be analytic on and within $\mathcal{L}$. Hence, when the nodes $0, \frac{[1]_{p, q}}{[n]_{p, q}}, \frac{[2]_{p, q}}{[n]_{p, q}}, \ldots, \frac{[k]_{p, q}}{[n]_{p, q}}$ are inside $\mathcal{L}$ and the pole $\alpha=p^{m} q^{-m}$ is outside, one has

$$
f\left[0, \frac{p^{n-1}[1]_{p, q}}{[n]_{p, q}}, \ldots, \frac{p^{n-r}[r]_{p, q}}{[n]_{p, q}}\right]=\frac{1}{2 \pi i} \oint_{\mathcal{L}} \frac{f_{m}(\zeta) d \zeta}{\zeta\left(\zeta-\frac{p^{n-1}[1]_{p, q}}{[n]_{p, q}}\right) \cdots\left(\zeta-\frac{p^{n-r}[r]_{p, q}}{[n]_{p, q}}\right)}
$$


By the residue theorem

$$
\begin{aligned}
f\left[0, \frac{p^{n-1}[1]_{p, q}}{[n]_{p, q}}, \ldots, \frac{p^{n-r}[r]_{p, q}}{[n]_{p, q}}\right] & =\sum_{j=0}^{k} \operatorname{Res}_{z=p^{n-j}} \frac{[j]_{p, q}}{[n] p, q} \frac{f_{m}(z)}{\prod_{j=0}^{k}\left(z-p^{n-j} \frac{[]_{p, q}}{[n]_{p, q}}\right)} \\
& =-\operatorname{Res}_{z=p^{m} q^{-m}} \frac{f_{m}(z)}{\prod_{j=0}^{k}\left(z-p^{n-j} \frac{[j]_{p, q}}{[n]_{p, q}}\right)} \\
& =-\frac{p^{-m(k+1)} q^{m(k+1)}}{\prod_{j=1}^{k}\left(1-p^{n-m-j} \frac{[j]_{p, q}}{[n]_{p, q}}\right)} .
\end{aligned}
$$

Since $f_{m}(z)=f_{m}(x)$ for $z=x \in[0,1]$, the statement follows from the divided difference representation (1.4).

Now, we find the asymptotic estimates for the coefficient $C_{k, n}^{p, q}$ in the next lemma.

Lemma 2.3 We have

$$
\lim _{n \rightarrow \infty} \prod_{k=1}^{n-j}\left(1-p^{n-m-j} q^{m} \frac{[k]_{p, q}}{[n]_{p, q}}\right)=\left(\frac{p^{2 j-m}}{q^{j-m}} ; \frac{p}{q}\right)_{\infty}
$$

for $j>m, q>p>1$.

Proof It is clear that

$$
\log \prod_{k=1}^{n-j}\left(1-p^{n-m-j} q^{m} \frac{[k]_{p, q}}{[n]_{p, q}}\right)=\sum_{k=j}^{n-1} \log \left(1-p^{n-m-j} q^{m} \frac{[n-k]_{p, q}}{[n]_{p, q}}\right)=\sum_{k=j}^{\infty} a_{k, n}^{p, q},
$$

where

$$
a_{k, n}^{p, q}= \begin{cases}\log \left(1-p^{n-m-j} q^{m} \frac{[n-k]_{p, q}}{[n]_{p, q}}\right), & k<n, \\ 0, & k \geq n .\end{cases}
$$

Since

$$
\begin{aligned}
\left|a_{k, n}^{p, q}\right| & \leq\left|\log \left(1-p^{n-m-j} q^{m} \frac{[n-k]_{p, q}}{[n]_{p, q}}\right)\right| \\
& \leq \frac{q}{q-p} \frac{p^{n-m-k} q^{m}[n-k]_{p, q}}{[n]_{p, q}} \leq \frac{q}{q-p} p^{n-k-m} q^{m},
\end{aligned}
$$

which gives $\sum_{k=j}^{\infty}\left|a_{k, n}^{p, q}\right|<\infty$, and by the Lebesgue dominated convergence theorem, we have

$$
\begin{aligned}
\lim _{n \rightarrow \infty} \sum_{k=j}^{n-1} \log \left(1-p^{n-m-j} q^{m} \frac{[n-k]_{p, q}}{[n]_{p, q}}\right) & =\sum_{k=j}^{\infty}\left(\lim _{n \rightarrow \infty} \log \left(1-p^{n-m-j} q^{m} \frac{[n-k]_{p, q}}{[n]_{p, q}}\right)\right) \\
& =\sum_{k=j}^{\infty} \lim _{n \rightarrow \infty}\left(1-p^{n-k-m} \frac{q^{m}}{q^{k}}\right),
\end{aligned}
$$


as a result

$$
\lim _{n \rightarrow \infty} \log \prod_{k=1}^{n-j}\left(1-p^{n-m-j} q^{m} \frac{[n-k]_{p, q}}{[n]_{p, q}}\right)=\log \prod_{k=j}^{\infty}\left(1-p^{n-k-m} \frac{q^{m}}{p^{k}}\right)
$$

which completes the proof.

The following lemma gives an upper bound for $n-m-1$.

Lemma 2.4 If $m \in \mathbb{N}, k=0,1,2, \ldots, n-m-1$, then

$$
\left|C_{k, n}^{p, q}\right| \leq \mathcal{C}_{m, p, q} p^{-m n} q^{m n}
$$

where $\mathcal{C}$ in $R H S$ is a positive constant, whose value need not to be addressed.

Proof For $n>m+1$ and from (2.3), we have

$$
\begin{aligned}
\left|C_{k, n}^{p, q}\right| & \leq \frac{p^{-m(k+1)} q^{m(k+1)}}{\left(1-\frac{p^{n-m-1} q^{m}[1]_{p, q}}{[n]_{p, q}}\right)\left(1-\frac{p^{n-m-2} q^{m}[2]_{p, q}}{[n]_{p, q}}\right) \cdots\left(1-\frac{p^{n-m-k} q^{m}[k]_{p, q}}{[n]_{p, q}}\right)} \\
& \leq \frac{p^{-m(n-m)} q^{m(n-m)}}{\left(1-\frac{p^{2(n-m-1)} q^{m}}{q^{n-1}}\right)\left(1-\frac{p^{2(n-m-2)} q^{m[2]_{p, q}}}{[n]_{p, q}}\right) \cdots\left(1-\frac{p^{n-m-k} q^{m}[k]_{p, q}}{[n]_{p, q}}\right)}, \\
\left|C_{k, n}^{p, q}\right| & \leq \frac{p^{-m n} q^{m n}}{p^{2 m} q^{2 m}\left(\frac{p}{q} ; \frac{p}{q}\right)_{\infty}} .
\end{aligned}
$$

Further, we discuss the nature of $C_{n-m+1, n}, \ldots, C_{n, n}$ as follows.

Lemma 2.5 For $m \in \mathbb{N}, q>p>1$,

$$
\left|C_{n-m, n}^{p, q}\right| \sim \mathcal{C}_{p, q, m} p^{-(m+1) n} q^{(m+1) n}, \quad n \rightarrow \infty
$$

Proof Using (2.3), we obtain the following

$$
\left|C_{n-m}^{p, q}\right|=\lambda_{n-m, n}^{p, q} \frac{p^{-m(n-m+1)} q^{m(n-m+1)}}{\left(1-\frac{p^{n-m-1} q^{m}[1]_{p, q}}{[n]_{p, q}}\right)\left(1-\frac{p^{n-m-2} q^{m}[2]_{p, q}}{[n]_{p, q}}\right) \cdots\left(1-\frac{p^{n-m-(n-m)} q^{m}[n-m]_{p, q}}{[n]_{p, q}}\right)} .
$$

From Lemma 2.3, we have

$$
\begin{aligned}
\left|C_{n-m}^{p, q}\right| & \sim \frac{\left(\frac{p^{m+1}}{q^{m+1}} ; \frac{p}{q}\right)_{\infty} q^{m(n-m+1)} p^{-m(n-m+1)} p^{m}\left(p^{n}-q^{n}\right)}{\left(\frac{p}{q} ; \frac{p}{q}\right)_{\infty} p^{n}\left(p^{m}-q^{m}\right)} \\
& \sim \frac{\left(\frac{p^{m+1}}{q^{m+1}} ; \frac{p}{q}\right)_{\infty} q^{m n} p^{-m n} p^{m}\left(p^{n}-q^{n}\right)}{\left(\frac{p}{q} ; \frac{p}{q}\right)_{\infty} q^{m(m-1)} p^{m(m-1)}\left(p^{m}-q^{m}\right)}, \\
C_{n-m, n}^{p, q} & =\mathcal{C}_{m}^{p, q} p^{-n(m+1)} q^{n(m+1)} .
\end{aligned}
$$

The nature of the remaining coefficients $C_{n-m+1, n}, \ldots, C_{n, n}$ is given as follows. 
Lemma 2.6 For $j=1,2, \ldots, m$, we have

$$
\lim _{n \rightarrow \infty} \frac{C_{n-m+j, n}^{p, q}}{C_{n-m, n}^{p, q}}=(-1)^{j}\left[\begin{array}{c}
m \\
j
\end{array}\right]_{p, q} p^{\frac{(n-j)(n-j-1)}{2}} q^{\frac{j(j-1)}{2}} .
$$

Proof Using (2.3) and (1.5), we get

$$
\begin{aligned}
& C_{n-m+j, n}^{p, q}=C_{n-m, n}^{p, q} \frac{\left(1-\frac{p^{m}[n-m]_{p, q}}{[n]_{p, q}}\right) \cdots\left(1-p^{m-j+1} \frac{[n-m+j-1]_{p, q}}{[n]_{p, q}}\right)}{\left(1-p^{-1} q^{m} \frac{[n-m+1]}{[n]_{p, q}}\right) \cdots\left(1-p^{-j} q^{m} \frac{[n-m+j]_{p, q}}{[n]_{p, q}}\right)} \\
& \lim _{n \rightarrow \infty} \frac{C_{n-m+j, n}^{p, q}}{C_{n-m, n}^{p, q}}=\frac{\left(1-\frac{p^{m}}{q^{m}}\right) \cdots\left(1-\frac{p^{m-j+1}}{q^{m-j+1}}\right)}{\left(1-\frac{q}{p}\right) \cdots\left(1-\frac{q^{j}}{p^{j}}\right)} \\
& \lim _{n \rightarrow \infty} \frac{C_{n-m+j, n}^{p, q}}{C_{n-m, n}^{p, q}}=(-1)^{j}\left[\begin{array}{c}
m \\
j
\end{array}\right]_{p, q} p^{\frac{(n-j)(n-j-1)}{2}} q^{\frac{j(j-1)}{2}} .
\end{aligned}
$$

Corollary 2.7 The following estimate holds:

$$
\left|C_{k, n}^{p, q}\right| \leq \mathcal{C}_{p, q, m} p^{-(m+1) n} q^{(m+1) n}, \quad k=0,1,2, \ldots, n,
$$

and $\mathcal{C}_{p, q, m}$ is independent of both $k$ and $n$.

Corollary 2.8 We have the following:

$$
\lim _{n \rightarrow \infty} \frac{C_{n-m, n}+\cdots+C_{n-m+j, n} x^{j}+\cdots+C_{n, n} x^{n}}{C_{n-m, n}}=(x ; p, q)_{m} .
$$

Proof The statement follows from Rothe's identity [6],

$$
(x ; p, q)_{m}=\sum_{j=0}^{m}(-1)^{j}\left[\begin{array}{c}
m \\
j
\end{array}\right]_{p, q} p^{\frac{(n-j)(n-j-1)}{2}} q^{\frac{j(j-1)}{2}} .
$$

\section{Main results}

- First we consider the case when pole $\alpha \in \mathbb{J}_{p, q} \backslash\{0,1\}$.

Now, we obtain the results that concern with the uniform approximation of $f_{m}(x), m \in \mathbb{N}$ by its $(p, q)$-Bernstein operators. It may be noted that, while the case when $\alpha \in[0,1] \backslash$ $\mathbb{J}_{p, q}$ can easily be examined by using the result and method of [27], the condition $\alpha \in \mathbb{J}_{p, q}$ requires a different approach.

Theorem 3.1 If $m \in \mathbb{N}$, then $B_{p, q}^{n}\left(f_{m} ; x\right) \rightarrow f_{m}(x)$ as $n \rightarrow \infty$ uniformly on any compact subset of $\left(-p^{(m+1)} q^{(m+1)}, p^{(m+1)} q^{(m+1)}\right)$.

Proof We consider the complex $(p, q)$-Bernstein operators given by

$$
B_{p, q}^{n}(f ; x)=\sum_{k=0}^{n} f\left(\frac{[k]_{p, q}}{p^{k-n}[n]_{p, q}}\right) p_{n, k}(p, q ; x), \quad n \in \mathbb{N}, z \in \mathbb{C},
$$


and the function $f_{m}(z)=\frac{1}{\left(z-p^{m} q^{-m}\right)}, z \in \mathbb{C}$. Let $n$ be large enough to satisfy the condition $\frac{[k]_{p, q}}{[n]_{p, q}} \neq p^{m} q^{-m}$. Then

$$
B_{p, q}^{n}\left(f_{m} ; z\right)=\sum_{k=0}^{n} C_{k, n}^{p, q} z^{k}
$$

where $C_{k, n}^{p, q}$ is given by (2.3). Let $\rho \in\left(0, p^{(m+1)} q^{-(m+1)}\right)$. Therefore for $|z| \leq \rho$ the following estimate is valid by Corollary 2.7 :

$$
\left|B_{p, q}^{n}\left(f_{m} ; z\right)\right| \leq \sum_{k=0}^{n}\left|C_{k, n}^{p, q} \rho^{k}\right| \leq \mathcal{C}_{p, q, m} \sum_{k=0}^{n}\left(p^{-(m+1)} q^{(m+1)} \rho\right)^{k} \leq \mathcal{C}_{p, q, m} \frac{1}{\left(1-p^{-(m+1)} q^{(m+1)} \rho\right)} .
$$

Hence it follows that the operators $\left\{B_{p, q}^{n}\left(f_{m}, z\right)\right\}$ are uniformly bounded in the disk $\{z$ : $|z| \leq \rho\}$ and convergent on the sequence $\left\{p^{j} q^{-j}\right\}_{j=m+2}^{\infty}$ having an accumulation point at 0 to the function $f_{m}(z)$ analytic in this disc. Using Vitali's convergence theorem, we have $B_{p, q}^{n}\left(f_{m} ; z\right) \rightarrow f_{m}(z)(n \rightarrow \infty)$ uniformly on any compact set in $\{z:|z| \leq \rho\}$ as $\rho \in$ $\left(0, p^{(m+1)} q^{-(m+1)}\right)$ was arbitrary. This completes the proof.

Next we demonstrate that, outside of the interval, operators diverge everywhere except a finite number of points.

Theorem 3.2 If $m \in \mathbb{N}$, then $\lim _{n \rightarrow \infty} B_{p, q}^{n}\left(f_{m} ; x\right)=\infty$ for $|x|>p^{(m+1)} q^{-(m+1)}, x \neq p^{(m+1)} \times$ $q^{-(m+1)}, x \neq p^{(m-1)} q^{-(m-1)}, x \neq p^{(m-2)} q^{-(m-2)}, \ldots, 1$.

Proof For exceptional points $p^{(m-1)} q^{-(m-1)}, p^{(m-2)} q^{-(m-2)}, \ldots, 1$, the situation has been analyzed in Lemma 2.1(a). We take $x$ satisfying $|x|>p^{(m-1)} q^{-(m-1)}$ different from these values. Let $n>m$ be sufficiently large such that (2.3) holds. By Lemma 2.4, we obtain

$$
\begin{aligned}
\left|\sum_{k=0}^{\infty} C_{k, n}^{p, q} x^{k}\right| & \leq \mathcal{C}_{m, p, q} \sum_{k=0}^{n-m-1} p^{-m k} q^{m k} x^{k}=\mathcal{C}_{m, p, q} \frac{\left(p^{-m} q^{m} x\right)^{n-m}-1}{p^{-m} q^{m} x-1} \\
& =o\left(\left(p^{-(m+1)} q^{(m+1)} x\right)^{n}\right), \quad n \rightarrow \infty .
\end{aligned}
$$

Hence

$$
\begin{aligned}
B_{p, q}^{n}\left(f_{m} ; x\right) & =\sum_{k=n-m}^{n} C_{k, n}^{p, q} x^{k}+o\left(\left(p^{-(m+1)} q^{(m+1)} x\right)^{n}\right) \\
& =C_{n-m}^{p, q} x^{n-m} g_{n}(x)+o\left(\left(p^{-(m+1)} q^{(m+1)} x\right)^{n}\right), \quad n \rightarrow \infty .
\end{aligned}
$$

By Lemma 2.5, $\left|C_{n-m}^{p, q}\right| \sim \mathcal{C}_{p, q, m}(x)\left(p^{-(m+1)} q^{(m+1)} x\right)^{n}$ as $n \rightarrow \infty$ whenever $|x|>p^{(m+1)} q^{-(m+1)}$, since $\lim _{n \rightarrow \infty} g_{n}(x)=(x ; p, q)_{m} \neq 0$, when $x \notin\left\{p^{(m+1)} q^{-(m+1)}, \ldots, 1\right\}$.

Lemma 3.1 Let $f_{0}$ be given by putting $m=0$ in (1.8). If $B_{p, q}^{n}\left(f_{0} ; x\right)=\sum_{k=0}^{n} C_{k, n}^{p, q} x^{k}$ then

$$
C_{k, n}^{p, q}=\frac{-1}{\left(1-p^{n-k} \frac{[k]_{p, q}}{[n]_{p, q}}\right)}, \quad k=0,1,2, \ldots, n-1, \quad C_{n, n}^{p, q}=a+\sum_{k=0}^{n-1} \frac{1}{\left(1-p^{n-k} \frac{[k]_{p, q}}{[n]_{p, q}}\right)}
$$


Proof For $k=0,1, \ldots, n-1$, on a specific choice of the contour $\mathcal{L}$, such that the nodes $0, \frac{[1]_{p, q}}{[n]_{p, q}}, \ldots, \frac{[k]_{p, q}}{[n]_{p, q}}$ are inside $\mathcal{L}$ while the pole $\alpha=1$ is outside, formula (2.4) implies

$$
C_{k, n}^{p, q}=\frac{-\lambda_{k, n}^{p, q}}{\prod_{j=0}^{k}\left(1-p^{n-j} \frac{[j]_{p, q}}{[n]}\right)}=\frac{-1}{\left(1-p^{n-k} \frac{[k]_{p, q}}{[n]_{p, q}}\right)}
$$

since by (1.3), $B_{p, q}^{n}\left(f_{0} ; 1\right)=f_{0}(1)=a$ and the statement is proved.

Corollary 3.2 For $k=0,1,2, \ldots, n-1$ with $q>p>1$ we have the following result:

$$
\left|C_{k, n}^{p, q}\right| \leq \frac{q}{q-p}
$$

- Now, we consider the case when pole $\alpha=1$.

Here the point of singularity $x=1$ is one of the nodes $\frac{[k]_{p, q}}{[n]_{p, q}}$. Consider the function $f_{0}$

$$
f_{0}(x)= \begin{cases}\frac{1}{x-1}, & x \in \mathbb{R} \backslash\{1\} \\ a, & x=1\end{cases}
$$

Theorem 3.3 If $f_{0}$ is given by (3.2), then the following holds:

(1) For all $x \in(-1,1]$,

$$
\lim _{n \rightarrow \infty} B_{p, q}^{n}\left(f_{0} ; x\right)=f_{0}(x)
$$

and the convergence is uniform on any compact subset of $(-1,1)$.

(2) For all $x \in \mathbb{R} \backslash(-1,1]$,

$$
\lim _{n \rightarrow \infty} B_{p, q}^{n}\left(f_{0} ; x\right)=\infty
$$

Proof (1) Since $B_{p, q}^{n}\left(f_{0} ; 1\right)=f_{0}(1)$, we need to prove only the uniform convergence of the compact subset of $(-1,1)$. For any $\rho \in(0,1)$ and $|z| \leq \rho$. From Corollary 3.2, we have

$$
\left|\sum_{k=0}^{n-1} C_{k, n}^{p, q} z^{k}\right| \leq \frac{\mathcal{C}_{p, q}}{1-\rho}
$$

Apart from that,

$$
\left|C_{n, n}^{p, q} z^{k}\right| \leq|a|+\sum_{k=0}^{n-1} \frac{1}{1-p^{n-k} \frac{[k]_{p, q}}{[n]_{p, q}}} \leq|a|+n\left|C_{k, n}^{p, q}\right| \leq|a|+\frac{n q}{q-p}
$$

whence

$$
\left|C_{n, n}^{p, q} z^{n}\right| \leq|a| \rho^{n}+\rho^{n} \frac{n q}{q-p} \leq \mathcal{C}_{p, q, \rho}
$$

Therefore, we conclude that the operators $B_{p, q}^{n}(f ; z)$ are uniformly bounded in any disk $\{z:|z| \leq \rho\}$ where $\rho \in(0,1)$. From Lemma 2.1(b) and Vitali's convergence theorem we arrive at our result. 
(2) Given that $x$ satisfies $|x|>1$, by Able's inequality, we have

$$
\left|\sum_{k=0}^{n-1} C_{k, n}^{p, q} x^{k}\right| \leq \frac{|x|^{n}-1}{|x|-1}\left(\left|C_{0, n}^{p, q}\right|+2\left|C_{n-1, n}^{p, q}\right|\right) \leq \frac{|x|^{n}}{|x|-1}\left(1+\frac{2 p}{p-q}\right)=\mathcal{C}_{p, q, x}|x|^{n}
$$

Meanwhile,

$$
\left|C_{n, n}^{p, q} x^{n}\right| \geq\left(\sum_{k=0}^{n-1} \frac{1}{1-p^{n-k} \frac{[k]_{p, q}}{[n]_{p, q}}}\right)|x|^{n}-|a| \cdot|x|^{n} \geq(n-|a|)|x|^{n} .
$$

Thus, $\left|B_{p, q}^{n}\left(f_{0} ; x\right)\right| \geq n|x|^{n}-\left(\mathcal{C}_{p, q, x}+|a|\right)|x|^{n} \rightarrow \infty$ as $n \rightarrow \infty$.

At $x=-1$, we have

$$
B_{p, q}^{n}\left(f_{0} ;-1\right)=\sum_{k=0}^{n-1} C_{k, n}^{p, q}(-1)^{k}+\left(a+\sum_{k=0}^{n-1} \frac{1}{1-p^{n-k} \frac{[k]_{p, q}}{[n]_{p, q}}}\right)(-1)^{n}
$$

and again applying Able's inequality,

$$
\left|\sum_{k=0}^{n-1} C_{k, n,}^{p, q}(-1)^{k}\right| \leq\left|C_{0, n}\right|+2\left|C_{n-1, n}\right| \leq 1+\frac{2 p}{p-q}
$$

On the other hand

$$
\left|\left(a+\sum_{k=0}^{n-1} \frac{1}{1-p^{n-k} \frac{[k]_{p, q}}{[n]_{p, q}}}\right)(-1)^{n}\right| \geq n-|a|
$$

which implies that

$$
\left|B_{p, q}^{n}\left(f_{0} ;-1\right)\right| \geq n-|a|-\left(1+\frac{2 p}{p-q}\right) \rightarrow \infty, \quad n \rightarrow \infty .
$$

Remark For justification of the statement that the extra parameter $p$ provides flexibility for approximation, one can see Remark 1 of [23].

Moreover, since for $q>p=1$ we recapture the $q$-Bernstein operators studied in [30], it is clear that the interval of uniform convergence for $B_{p, q}^{n}$ in Theorem 3.1, i.e. $\left(-p^{m+1} q^{m+1}, p^{m+1} q^{m+1}\right)$, is larger than the interval of uniform convergence $\left(-q^{m+1}, q^{m+1}\right)$, obtained by Theorem 2.1 in [30].

Funding

The third author would like to thank "Prince Sultan University for funding this work through research group Nonlinear Analysis Methods in Applied Mathematics (NAMAM)" group number RG-DES-2017-01-17.

\section{Competing interests}

The authors declare that they have no competing interests.

\section{Authors' contributions}

All the authors contributed equally and significantly in writing this paper. All of them read and approved the final manuscript.

\section{Author details}

'Department of Mathematics, Aligarh Muslim University, Aligarh, India. ²Department of Applied Mathematics, Aligarh Muslim University, Aligarh, India. ${ }^{3}$ Department of Mathematics and General Sciences, Prince Sultan University, Riyadh, Saudi Arabia. 


\section{Publisher's Note}

Springer Nature remains neutral with regard to jurisdictional claims in published maps and institutional affiliations.

\section{Received: 4 February 2019 Accepted: 3 May 2019 Published online: 14 May 2019}

\section{References}

1. Acar, T., Aral, A., Mohiuddine, S.A.: On Kantorovich modification of (p, q)-Baskakov operators. J. Inequal. Appl. 2016, 98 (2016)

2. Acar, T., Aral, A., Mohiuddine, S.A.: Approximation by bivariate ( $p, q)$-Bernstein-Kantorovich operators. Iran. J. Sci. Technol., Trans. A, Sci. 42(2), 655-662 (2018)

3. Acar, T., Aral, A., Mohiuddine, S.A.: On Kantorovich modification of ( $p, q)$-Bernstein operators. Iran. J. Sci. Technol, Trans. Sci. 42, 1459-1464 (2018)

4. Acar, T., Aral, A., Mursaleen, M.: Approximation by Baskakov-Durrmeyer operators based on $(p, q)$-integers. Math. Slovaca 68(4), 897-906 (2018)

5. Acar, T., Mohiuddine, S.A., Mursaleen, M.: Approximation by ( $(p, q)$-Baskakov-Durrmeyer-Stancu operators. Complex Anal. Oper. Theory 12(6), 1453-1468 (2018)

6. Andrews, G.E., Askey, R., Roy, R.: Special Functions. Cambridge University Press, Cambridge (1999)

7. Bernstein, S.N.: Démonstration du théoréme de Weierstrass fondeé sue la calcul des probabilités. Commun. Soc. Math. Charkaw 213, 1-2 (1992)

8. Bin Jebreen, H., Mursaleen, M., Naaz, A.: Approximation by quaternion $(p, q)$-Bernstein polynomials and Voronovskaja type result on compact disk. Adv. Differ. Equ. 2018, 448 (2018)

9. Bohman, H.: On approximation of continuous and of analytic functions. Ark. Mat. 2(1), 43-56 (1995)

10. Gal, S.G.: Approximation by complex $q$-Lorentz polynomial, $q>1$. Mathematica 54(77), 53-63 (2012)

11. Kadak, U.: On weighted statistical convergence based on $(p, q)$-integers and related approximation theorems for functions of two variables. J. Math. Anal. Appl. 443(2), 752-764 (2016)

12. Kadak, $\mathrm{U}$ : Weighted statistical convergence based on generalized difference operator involving $(p, q)$-Gamma function and its applications to approximation theorems. J. Math. Anal. Appl. 448(2), 1633-1650 (2017)

13. Kadak, U., Mishra, V.N., Pandey, S.: Chlodowsky type generalization of $(p, q)$-Szász operators involving Brenke type polynomials. Rev. R. Acad. Cienc. Exactas Fís. Nat., Ser. A Mat. 112(4), 1443-1462 (2018)

14. Kadak, U., Mohiuddine, S.A.: Generalized statistically almost convergence based on the difference operator which includes the ( $p, q)$-gamma function and related approximation theorems. Results Math. 73, Article ID 9 (2018)

15. Khan, A., Sharma, V.: Statistical approximation by (p,q)-analogue of Bernstein-Stancu operators. Azerb. J. Math. 8(2), 100-121 (2018)

16. Korovkin, P.P.: Convergence of linear positive operator in the space of continuous function. Dokl. Akad. Nauk SSSR 90, 961-964 (1953)

17. Lorentz, G.G.: Bernstein Polynomials, 2nd edn. Chelsea, New York (1986)

18. Lupaş, A.: A q-analogue of the Bernstein operators. In: Seminar on Numerical and Statistical Calculus, pp. 85-92 (1987)

19. Mishra, V.N., Mursaleen, M., Pandey, S., Alotaibi, A.: Approximation properties of Chlodowsky variant of (p,q)-Bernstein-Stancu-Schurer operators. J. Inequal. Appl. 2017, 176 (2017)

20. Mishra, V.N., Pandey, S.: On (p, q)-Baskakov-Durrmeyer-Stancu operators. Adv. Appl. Clifford Algebras 27(2), 1633-1646 (2017)

21. Mohiuddine, S.A., Acar, T., Alotaibi, A.: Durrmeyer type $(p, q)$-Baskakov operators preserving linear functions. J. Math. Inequal. 12, 961-973 (2018)

22. Mursaleen, M., Ansari, K.J., Khan, A.: On (p, q)-analogue of Bernstein operators. Appl. Math. Comput. 266, 874-882 (2015) [Erratum: Appl. Math. Comput., 278 (2016) 70-71]

23. Mursaleen, M., Khan, F., Khan, A.: Approximation by $(p, q)$-Lorentz polynomial on a compact disk. Complex Anal. Oper. Theory 10(8), 1725-1740 (2016)

24. Mursaleen, M., Naaz, A., Khan, A.: Improved approximation and error estimations by King type (p, q)-Szasz-Mirakjan-Kantorovich operators. Appl. Math. Comput. 348, 175-185 (2019)

25. Mursaleen, M., Nasiruzzaman, Md., Khan, F., Khan, A.: $(p, q)$-analogue of divided difference and Bernstein operators. J. Nonlinear Funct. Anal. 2017, Article ID 25 (2017)

26. Mursaleen, M., Rahman, S., Alkhaldi, A.H.: Convergence of iterates of $q$-Bernstein and $(p, q)$-Bernstein operators and the Kelisky-Rivlin type theorem. Filomat 32(12), 4351-4364 (2018)

27. Ostrovska, S.: q-Bernstein polynomial and their iterates. J. Approx. Theory 123, 232-255 (2003)

28. Ostrovska, S.: q-Bernstein polynomial of the Cauchy kernel. Appl. Math. Comput. 198(1), 261-270 (2008)

29. Ostrovska, S., Özban, A.Y.: On the $q$-Bernstein polynomial of unbounded function with $q>1$. Abstr. Appl. Anal. 2013 , Article ID 349156 (2013)

30. Ostrovska, S., Özban, A.Y.: The $q$-Bernstein polynomials of the Cauchy kernel with a pole on $[0,1]$ in the case $q>1$. Appl. Math. Comput. 220, 735-747 (2013)

31. Phillips, G.M.: Bernstein polynomials based on the 9 -integers. Ann. Numer. Math. 4, 511-518 (1997)

32. Srivastava, H.M., Ozer, F., Mohiuddine, S.A.: Construction of Stancu-type Bernstein operators based on Bézier bases with shape parameter $\lambda$. Symmetry 11(3), Article ID 316 (2019) 